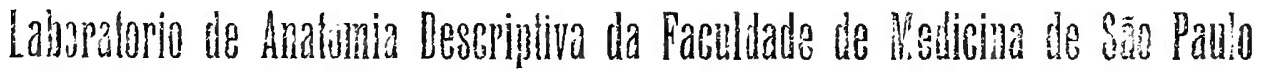

\title{
DIRIGIDO PELO
}

\author{
PROF. A. BOVERO
}

\section{Duas Anomalias Musculares}

\section{(M. Chonciro-epitrochleano e M. Accessorio da Cavidade Orbitaria)}

Observações de PAUlO SAWAYA, ODORICO MACHADO DE SOUSA, JOÃO THOMAZ DE AQUINO E JORGE F. SAINATI

Durante os trabalhos praticos de anatomia, realisados em Abril deste anno (1925), quando dissecavamos, primeiramente, a porção axiliar do plexo brachial e depois os musculos e nervo's da cavidade orbitaria, do mesmo cadaver, (n. ${ }^{\circ} 1354$, E .O., mulher parda, 40 annos, fallecida a 25-IX-924 por tuberculose pulmonar). tivemos a opportunidade de encontrar, em ambos os lados, duas formações musculares anomalas, que motivaram esta breve exposição, á qual damos o feitio de um simples relatorio de sala de dissecção, visto tratar-se de anomalias musculares conhecidas na literatura anatomica.

\section{MUSCULO CHONDRO-EPITROCHLEANO}

A formação muscular anomala que encoratramos, quando dissecámos a porção axillar do plexo brachial, é bilateral, e extende-se da região thoraxica, atravez da axillar, até a porção distal da região brachial anterior.

De ambos os lados o $\mathrm{m}$. grande peitoral se apresenta relativamente delgado, fraco no seu desenvolvimento; a sua porção infero-lateral é representada por uma faixa muscular que abandonando o restante do 
musculo, na parte lateral da parede axillar anterior, descreve uma curva de concavidade voltada para baixo, para se dirigir á região antero-medial do braço, attingindo a epitrochlea.

Esta formação anomala corresponde ao musculo chondro-epitrochleano descripto primeiramente por Duvernoy mo chimpanzé e depois por Wood (1860), muitissimas vezes no homem. Delle se encontram diffưsas descripções não somente na casuistica, espalhada ım jornaes anatomicos, mas principalmente nos tratados de anomalias musculares, como nos de Testut (1884), Le Double (1897) e, mais recentemente, na classica monographia de Eisler (1912), sendo tambem citado na maioria dos tratados classicos de anatomia descriptiva, que fazem referencias ás anomalias musculares ( $T$ estut, Quain, Romiti, Poirier, Cunningham, Piersol, Rauber-Kopsch e outros).

Se bem que as formações que têm o nome de $\mathrm{m}$. chondro-epitrochleano, nas suas differentes modalidades e graos de desenvolvimento, sejam largamente discutidas e a sua casuistica seja numerosa, achamos opportuna a sua descripção, por ser bilateral, porque á esquerda está em relação com uma expansão da fascia brachial, que se comporta como um arco axillar membranoso, juntando-se depois ao septo intermuscular intermo do braço; e emfim ,muito particularmente, porque tivemos a fortuna de evidenciar, com muita minucia, a sua innervação, sendo o nosso achado facilitado pelo assumpto inicial da nossa dissecção (plexo brachial). Nesse ponto acreditamos ser o caso, provavelmente, novo na literatura, de modo que a demonstração minuciosa da innervação justifica, por si mesma, a razão desta breve nota.

Esta exposição foi baseada essencialmente nas obras acima lembradas, visto ser muito difficil em o nosso meio o conhecimento dos casos registrados na literatura, dada a falta quasi completa de collecções de jornaes de anatomia contendo as respectivas descripcõoes.

Desejamos salientar que, para o conhecimento do m. ch.-ep., se encontra uma valiosissima contribuição na literatura portugueza, pois, entre as publicações de $H$. de Vilhena $(19.14,1921)$, Pires de Lima (1914), H. Bastos Monteiro (1920, 1921) e A. Tavares (1924). algumas são, de facto, não, somente as mais recentes, mas tambom as quie apresentam maior numero e aspectos mais variados do referido $\mathrm{m}$. 
E' a esta serie de publicações portuguezas, que podem recorrer aquelles que desejarem ter uma noção de conjuncto, não só no que diz respeito ás differentes modalidades do m. ch.-ep., como ás questões ligadas á sua interpretação morphologica.

São estas as razões que nos levaram a abstermos de um exame critico pormenorizado da literatura sobre o assumpto, o que aliás nos obrigaria a repetir essencialmente, 0 , que se encontra em Testut, Le Double, $E_{i}$ isler, etc., e nas referidas publicações portuguesas, e a limitarmos a uma simples illustração do nosso caso.

Além dos numerosos casos, citados pelos ditos AA., apresentam-se outro's de Tenchini (1880), Bianchi (1886), Tobler (1902), Böse (1904), e Lunghetti (1906) os quaes, ou não foram mencionados pelos referidos AA., ou são publicações posteriores aos tratados de Testut e Le Double.

Particularmente interessantes são os casos de Griffith (1899), que abservou o m. ch.-ep. no vivo, e de Pires de Lima (1916) que, no cadaver, poude diagnosticar o m. aromalo antes da dissecção.

Menção especial deve ter a observação de Pickler (1916) que verificou, no vivo, o m. ch.-ep. juntamente com o arco axillar muscular; essa mesma associação foie ncontrada, no cadaver, por Tobler e Böse.

Entre nós, embora tenha sido estudado cuidadosamente o arco axillar muscular, no cadaver (David - 1921, Lindenberg Quintanilha 1923), e no vivo (David), não temos noticia de illustrações de casos do m. ch. -ep.

Tiramos, como simples curiosidade, mas com algumas reservas, do tratado de Le Double, as multiplas denominações com as quaes esse $\mathrm{m}$. anomalo é indicado pelos AA.: Humero-abdominalis (Klein), Abdomino-humeral (Dugès), Chondro-epitrochlearis (Wood), Brachioabdominalis (Zenker), Brachio-lateralis (Humphry), Portio abdominalis pectoralis majoris (His), $3 .^{\circ}$ peitoral (Broca e Pozzi), Pectoralis quartus (Haughton e Macalister), Portio pyramidalis pectoralis majoris (Rolleston e Galton), Feixe supplementar do grande peitoral (Chudzinshi), Abdomino anti-brachial (Testut), Costo-humeralis (Huxley), Epigastric slip (Perrin). 
E' sufficiente esta enumeração para comprehender-^e como muitas dessas denominações não se refiram e nem possam referir-se ao verdadeiro m. ch.-ep., seja este rudimentar ou no maximo desenvolvimento, mas indiquem nada mais que a porção abdominal do $\mathrm{m}$. grande peitoral.

INSERÇÃO E RELAÇÕES: - Já dissemos quie o m.ch.-ep. do nosso caso é bilateral; o seu comportamento é identica de ambos os lados, apenas com algumas variações.

A' direita a formação anomala tem a sua origem na extremidade medial da 4.a e 5.a cartilagans costaes, e espaço intercostal correspondente, por meio de feixes musculares d́elgados e pallidos, cujas inserções mediaes e inferiores se perdem numa delgada faixa que se confunde, caudalmente, com a bainha de contenção dos $\mathrm{mm}$. grande obliquo e recto anterior do abdomen.

Esta porção do m. ch. ẹp., que corresponde á porção caudal do m. grande peitorial (porção costo-abdominal), de côr pallida, está disposta em forma de leque, muito achatado, cujos feixes convergem para cima e para fora, sobrepondo-se superficialmente, pelo seu bordo superior, á parie mais baixa da porção costal propriamerute cita do merno musculo.

Medialmente á linha mamillar tem-se a impressão de que esta porção inferior do m. grande peitoral se soprepõe, como uma camada superficial, á porção de origem costal mais distal do m. ch.-ep. e de terminação no labio externo da corrediça bicipital. Esta sobreposição é marcada por um intersticio fissural, que a dissecção poz en evidencia.

Logo lateralmente á linha hemiclavear os feixes musculares da formação, anomala pela sua termiração, separam se da poçção costa propriamente dita do $\mathrm{m}$. grande peitoral, dirigindo-se para baixo e la le:a!mente, emquanto que a outra se dirige, como de habito, para a corrediça bicipital, onde se insere, sendo recoberta ahi, na sua porção terminal, pelo tendão da porção estermal do grande peitoral.

O feixe ch.-ep., pouco antes de se afastar da porção costal do m. grasade peitoral, tem a forma de uma fita, achatada em sentido medio-lateral, e está comprehendida na parte mais caudal do pilar anterior da axilla, da qual delimita a curva que, no individuo antes da dissecção, devia ser mais accentuada e mais exideñte que norma mente. 
Dirige-se depois para baixo, para a região brachial anterior, conservando-se logo infrapcnevrotico. Nestas condições, continúa muscular até a união do terço irferior com os dois terços superires do braço, onde se lança em um tendão delgado e achatado, que ešć em relação de perfeita continuidade com a aponevrcse de contenção cóos mm. da loja anterior do braço, ligando-se ao septo intermuscular interno.

$A^{\prime}$ esquerda o m. anomalo apr`senta, como já foi d’ to, o mesmo, conjuncio de disposições, differindo somente em que, na sua origem, o m. apparece mais roibusto e tem a sua inserção mais baixa que á dirieta, isto é, alguns feixes musculares attingem $0,5 .^{\circ}$ espaço intercostal, ao nivel do qua! é mais evidente a continuação com a bainha do $\mathrm{m}$. grande obliquo. Além disso, os feixes que constituem o m. ch.-ep. não apparecem sobrepostos, mas sim simplesmente contiguos, sendo separados, por um intersticio linear, dos feixes mais distaes da porção costal propriamente dita. Por isso, o afastamento do feixe chondro-abdominal da porção costal do $\mathrm{m}$. grande peitoral, lateralmente á linha hemiclavear, se faz á custa de uma simples ampliação desta interlinha.

$\mathrm{Na}$ terminação brachial, os feixes musculares d’o m. ch.-ep. esquerdo se lançam, no terço medio do braço, num lendãosinho laminar, nacarado, de $3 \mathrm{~mm}$. de largura, á maneira dos mm. cemi-penniformes e do qual examinaremos separadamente a porção upericr de origem, e a sua terminação.

Cranea!mente se origina, sob a face profunda de uma expansão aponevrotica, que o tendão termina: da porção costal do $\mathrm{m}$. grande peitoral envia á fascia brachial, logo antes de inse:ir-se no labio lateral da corrediça bicipital. Resulta das di.pozições desta exijansão aponevrotica uma verdadeira bainha de cortenção, que recobre o feixa anomalo e o feixe vasculo nervoso do braço.

Este tendãosinho que apparece, como dissemos, na face profunda desta expansão, e cuja individualisação pode ser verificada muito alta, na superficie profunda do mesmo tendão te:minal da porção costal do $\mathrm{m}$. grande peitoral, recebe no seu borclo anterior, na parte media do braço e por uma extensão de cerca 4 cms., os feixes musculares do ch.-ep., apparecendo, até este ponto, iso'ados do septo inter-muscular interno. 
A coms. 3,5 para cima da epitrochlea, funde-se com este mesmo septo, do qual representa depois o bordo ventral muito saliente e de apparencia realmente tendinosa. Nesta sua parte inferior, constiture o tendão terminal do m. ch.-ep.

A' direita, entretanto, os seus feixes musculares se lançam directamente num tendão terminal, muito curto, sem o aspecto semi-penniforme descripto á esiquerda, o qual se conserva isolado somente por uma extensão de cms. 1,5 e depois, a 9 cms. da epitrochlea se funde intimamente com o septo intermuscular interno, occupanío, como do lado crposto, o bordo ventral até a epitrochlea.

No percurso axillo-brachial o $\mathrm{m}$. anomalo decorre, em ambos os lados, no bordo anterior da base da axilla, cruzando por diante o feixe vasculo nervoso do braço, chegando até o bordo medial da curta p̧orção da biceps; dahi para baixo acha-se anterior e lateralmente ao n. mediano, arteria e veias humeraes.

Relativamente ás relações do $\mathrm{m}$. ch-ep. com os vasos humeraes são dignas de notar-se, pelo interesse cirurgico, as observações de Testut (1892). Este A. nota que não haveria algum obstaculo á ligadura da arteria humeral, na região anterior do braço, quando o m. ch."ep. nesta região é representado somente por ùm tendão que cruza obliquamente a referida arteria. O caso será diverso quando se tratar de um corpo muscular desenvolvido recobrindo a arteria humeral, surprehendendo assim o cirurgião. Em taes casos, como para o arco axillar muscular, pode ser necessario, para attingir a arteria, deslocar o m. ou mesmo secciona-lo transviersalmente. 'Provavelmente isto teria acontecido no nosso caso, numa eventual ligadura da arteria humeral na sua parte alta.

O nervo cutaneo medial do ante-braço acompanhado pela veia basilica, perfura a aponevrose na parte media do braço, passando por diante do m. anomalo immediatamente acima da porção isolada do seu tendão terminal.

INNERVIAÇÃO: - Conseguimos patentear, como dissemos, com minudencia ,a innervação do m. ch.ep., por nós dissecado, o que aliás foi facilitado pelo objecto inicial da nossa dissecção.

Assim é que, ao nivel da linha axillar anterior, na altura do 4. 'espaço intercostal, o m. anomalo, ahi em forma de fita, é atravessado pelo 
ramo perfurante lateral do $4 .^{\circ}$ espaço que, depois de the ceder a!guns filetes, vae distribuir-se na pelle da região mamaria. $\mathrm{O}$. ch. ep. recebe tambem filetes do $3 .^{\circ}$ perfurante lateral, evidenciado somente á esquerda, não sendo possivel demonstra-lo á direita por ter sido, provavelmente, seccionado. Onde o: $\mathrm{m}$. faz a curva da axilla apresenta uma fenda, muito eviderite á direita, pela qual corre o ramo perfurante lateral do $2 .^{\circ}$ espaço. Nesta passagem do nervo o $\mathrm{m}$. forma uma botoeira, cuja porção caudal é muito mais delgada que a porção craneal.

Além disso, os nn. da formação plexiforme constituida pelos nn. thoraxicos anteriores dão origem ao ramo superficial do pequeno $n$. thoraxico anterior, que se subdivide dando um ramo para a porção costal do m. grande peitoral e um outro ramusculo para o ch.-ep. sob cuja face profunda decorrem os filetes nervosos que nelle penetram ao nivel da 4.a costella.

Não nos foi possivel evidenciar, no braço, filettes nervosos procedentes dos ramos terminaes do plexo.

Em resumo, o m. anomalo, de ambos os lados, tira a sua innervação do plexo accessorio ventral (C5) C6 - C7 (C8) (T1) (Schumacher), constituido pelos nn. thoraxicos anteriores, e dos perfurantes lateraes do $2 .^{\circ}, 3 .^{\circ}$ e $4 .^{\circ}$ espaços.

\section{II}

\section{MUISOULO ACCESSORIO DA CAVIDADE ORBITARIA}

O musculo considerado como anomalo foi observado nas duas cavidades orbitarias, com pequemas differenças á direita e á esquerda. Trata-se de um feixinho carnoso, de uma largura maxima de $2 \mathrm{mms}$. e de menos de $1 \mathrm{~mm}$. de espessura, que se origina, porteriormente, junto ao $\mathrm{m}$ elevador da palpebra superior, no contorno superio: do annel de Zinn e deco:rendo para diante, ao longo do angulo supero-medial da cavidade orbitaria, vae terminar de modo um pouco diverso nos dois lados. 
$\mathrm{Na}$ orbita direita o m. elevador da palpebra sup. apresenta-se bastante atrophiado, sendo quasi igual em tamanho ao feixe anomalo. $O$ feixe que o representa tem a direcção habitual, para frente e um pouco para fóra, delimitando com o feixe anomalo um angulo de cerca de $40^{\circ}$. Nas suas relações e na sua inserção pa'pebral, não ha particularidade a salientar-se. O feixe muscular anomalo, logo atraz da trochlea do $\mathrm{m}$. grande obliquo, se continúa por feixes tendinosos terminaes, que passam em baixo da mesma, sem contrahir, com a polia. relações de continuidade. Ahi elles se dividem em dois fasciculos: um medial e um lateral. O fasciculo medial, mais robusto, vae inserir-se, atravez da capsula fibrosa na parte mais anterior da superficie lateral do processo orbitario do frontal, a $3 \mathrm{~mm}$ abaixo da inserção da polia de reflexão do $\mathrm{m}$. grande abliquo. $\mathrm{O}$ f. lateral, delgadissimo, atravessa o septo orbitario e se resolve numa lamina extremamente fina que vae unir na espessura da palpebra superior. á expansão medial do tendão do m. elevador da palpebra sup. Entre estes dois feixes terminaes decorre o ramo superior do nervo nasal.

$\mathrm{Na}$ orbita esquerda o m. elev. da palpebra sup. tem o seu tamarho normal e a largura da sua parte media é de $5 \mathrm{mms}$. As inserções do tendão termiral do feixe accessorio se fazem um pouco differente neste lado, isto é, por um fasciculo medial, que se insere tambem na superficie orbitaria do frontal, mais perto porem da espinha trochlear ( $1 \mathrm{~mm}$ inferiormente); por um $\mathrm{f}$. lateral que dá uma porção principal, media do tendão terminal do $m$. accessorio, que se continua, claramente, com o contorno posterior da trochea cartiliginosa, e por uma terminação palpebral que se comporta como do lado opposto.

A differença entre o comportamento do $\mathrm{m}$. dos dois lados, está precisamente nesta estricta relação, existente somente á esquerda, com a polia de reflexão do $\mathrm{m}$. grande obliquo.

Em ambos os lados, o m. accessorio, na sua inserção de origem no annel de Zinn, é recoberto, parcialmente, pelo m. elev. da palpebra sup., estando logo depois, situado medialmente á origem do m. grande obliquo. No seu percurso está situado medialmente aos dois referidos 
mm., sendo costeado inferiormente pelo bordo superior do m. recto interno, tendo ,como estes, as habituaes nelações com a bola gordurosa da orbita.

\section{*}

Para a interpretação desse feixe accessorio é util recorrer-se ao estudo especial, não muito claro, que deste $\mathrm{m}$. fez Le Double, ou aos estudos das anomalias musculares da cavidade orbitaria, nos tratados clas sicos de Testu, Charpy, etc.

Não ha muita clareza nos AA. na descriminação dos differentes mm. accessorios da cavidade orbitaria, de modo que, ao nosso m. podem ser, indifferentemente, dadas varias das denominações utilisadas pelos diversos AA. para indicar disposições singulares. Assim, si considerarmos a atrophia do m. elev. da palpebra sup., á direita, concomitante á existencia de um feixe supranumerario, como tambem a terminação parcial deste na espessura da palpebra superior, poderiamos interpretar o $\mathrm{m}$. por nós descripto, como sendo um feixe accessorio do $\mathrm{m}$.elevador da palpebra sup., simulando um $m$. elevador duplo. Oui, considerando o percurso mais ou menos parallelo á porção directa do $\mathrm{m}$. grande obliquo, como $m$. comes obliquì superioris de Albinus.

Levando em conta ${ }_{i}$ seu tamanho e sua pequena espessura, seria apropriada a denominação de gracillimus orbitis de Bochdaleck (1868).

Firnalmente, pela verificação de sua terminação principal, embora somente á esquerda, no contorno posterior da trochlea cartilaginosa, seria natural interpreta-lo como sendo um caso do musculas tensor trochleæ, visto por Albinus, Boldrini (1669), Kuimus (1724), e particularmelnte por Budge (1859) que o considerou como uma formação normal, tendo encontrado 15 vezes em 20 individuos. Entretanto, H. Virchow o teria visto somente uma vez em muitos individuos. 
O nosso caso não tem certamente a pretenção de alguma novidade. É notavel porém ,a opportunidade de sua descripção, accérituada pela observação precedente, por ser uma indicação util para pesquizas systematicas tendentes a coordenar e interpretar fornações provavelmente muitas vezes identicas, apontadas com romes differervies.

\section{BIBLIOGRAPUA}

\section{I - MUSCULO CHONDRO-EPITROCHLEANO}

1) BIANAHI S. - Varictá muscolari. Lo "Sperimentale". Firenze. 1886.

2) DAVID (FILFiO) J. - Contribuição ao estudo do arco axillar muscular. These. Bahia, 1921.

3) EISLER P. - Die Musheln des Stammes. Bardeleben's Handbuch d. Anat. des Menschen. 1912. Bd. 2, Abt. 2. ${ }^{\circ}$, th. 1, S. $461-464$.

4) CRUBER W. - Ueber einen besonderen $\mathrm{K}_{\text {anal für den Nervus }}$ medianus im sulcus bicipitales internus bei Vorkcmmicin eines Musculus costo-epitrochlearis. Archiv f. Anat. u. Physiol. Anat. Abthlg. Jahrg.

1877 - S. 411 - 423. Resumo em Jahresberichte über die fortschritte der Anatomie und Physiologie. Bd. VII. (Literatur 1878) Erste Abtheilung: Anat. 1879. S. 196-197.

5) HUNTINGTON G. S. - The derivation and significance of supernumerary muscles in the pectoral region. Journal of Anat. and Physiol. October 1914. vol. XXXIX.

6) LE DOUUbL A. F. - Traité des variations du système musculaire de l'homme et de leur signification au point de vue de l'anthropologie zoologique. 1897. T. I. p. 248.

7) LINDENBERG QUINTANILHA I. - Arco axillar muscular. Revista de Medicina do Cantro Academico Oswalço Cruz. Vol. VII. n. 28 , pg. 3-4. 
8) LUNGHETTI B. - Sopra un muscolo sopranumerario axillo-epitrochleare e su altre anomalie muscolari. Atti della R. Academia dei Fisiocritici. 1906. Serie IV. Vol. XVII.

9) MONTEIRO (BASTOS) H. - Notas anatomicas, (XXXIVXLVII). Anaes da Faculdade de Medicina do Rio de Janeiro. 1921. Ano IV .pg. 250.

9-a) IDEM - Sur le muscle chondro-épitrochlearis. Sur les muscles periclaviculaires surnumeraires. Bulletin de la Société Portugaise des Sciences Naturelles, 1921. T. IX.

10) PERRIN J. B. - Notes on some variations of the pectoralis maj. with its associate muscles. Resumo do Bericht ueber die Fortschritttet des Anat. und Physiol. 1870. S. 233.

11) PICKLER K. - Achselbogen und M. chondro-epitrochlearis bei dernselben Träger. Anatomische Anzeiger. 1916. Bd. 49 п. ${ }^{\circ} 14$ S. 383.

12) PIRES DE LIMA J. A. - Do.s casos de m. chondro-epitrochlear. Archivo de Anat. e Anthropologia, 1914. Vol. I n. 3, pag. 234.

13) TAVARES A. J. - Estudos sobre as variações muscuiares do thorax 1924. pg. 91-95.

13-a) IDEM - Faisceau Musculaire Sterno-chondro-épitrochléen. Comptes Rendus de l'Association des Anatcm.stes. 1924. og. 242.

14) TEIXEIRA BASTIOS - Musculo chontromptrochicano. Anais Scientificos da Faculdade de Medicina do Porto. 1917-18. Vol. IV. n. ${ }^{\circ} 1$ pg. 155.

15) TENCHINI L. - Di un nuovo Muscolo Sopranumerario (costocmerale) del Braccio Umano. Bolletino Scientifico. Pavia. 1980.

16) TESTUT L. - Les anomalies musculaires chez l'Homme expliquées para l'Anatomic comparée, leur importance en Anihropologie Paris, 1884. pg. 18-28.

16-a) IDEM - Les anomalies musculaires considerées au point de vue de la ligature des artères. Paris. 1392. pg. 21-23. 
17) VILHENA (de) H. - Musculo supra-numerario Chondro-epitrochleano. Arch. de Anat. e Anthropol. Lisboa. 1913-14. Vol I. pg. 24.

17-a) IDEM - Tendães pectoro-epitrochleanos e feixes ou $\mathrm{mm}$. condro é costo-epitrochlcanos. Ibid. Lisboa 1921. Vol. VII. n. 2. pg. 89.

\section{II - MUSCULO ACCESSORIO DA CAVIDADE ORBITARIA}

1) BOCHDALECK. - Beitrag zu den anomalen Muskeln der Augenhohle. Prager Vierteljahrsschr. Bericht ueber die Fortzritte der Anat. und Physiol. 1868. Bd. IV, p. I. S. 123.

2) BUDGE. - Beschreibung eines neuen Muskels und mehrerer Muskelun Knochwarietäten-Zeistschrift für ration. Medic. Ibid: 1859. Bd. VII, Heft 2. S. 278. Resumo em Bericht über die Fortschritte der Anatomie und Physiologie im Jahre 1859.-1861 pg. 165.

3) CADIARSO A. R., et Goyanes J. J. B. - Sur un faisceau "surnuméraire du releveur de la paupière supérieure. Bulletins et Mémoires de la Societé Arratomique de Paris. 1925. N. 8. 95.e année. pgs. 242-243.

4) LE DOUBLE A. F. - Traté des variations du système musculaire de l'Hommie et de leur signification au point de vue de l'antropologie zoologique. Paris. 1897. T. I. pg. 48-50.

4-a) IDEM - De la possibilité du dévelopement dans l'espece humaine du m. oblique superieur de l'oeil des vertébrés inferieurs á l'ordre des mamifères. Bibliographie Anatomique. Paris, 1901. T. IV. Fasc. 1.

\section{ANUNCIEM na "REVISTA de MEDICINA"}

MEOIANTE PEDIDO ENVIAMOS TABELAS DE PREÇOS $[$ PRE\&-

TAMOS PRONTAMENTE QUAESQUIR CUIRAS INFORDIAÇŌES 